\title{
FORGe: prioritizing variants for graph genomes
}

\author{
Jacob Pritt ${ }^{1,2}$, Nae-Chyun Chen ${ }^{1,2}$ and Ben Langmead ${ }^{1,2^{*}}$ (D)
}

\begin{abstract}
There is growing interest in using genetic variants to augment the reference genome into a graph genome, with alternative sequences, to improve read alignment accuracy and reduce allelic bias. While adding a variant has the positive effect of removing an undesirable alignment score penalty, it also increases both the ambiguity of the reference genome and the cost of storing and querying the genome index. We introduce methods and a software tool called FORGe for modeling these effects and prioritizing variants accordingly. We show that FORGe enables a range of advantageous and measurable trade-offs between accuracy and computational overhead.
\end{abstract}

\section{Introduction}

Assembled genomes are typically stored and understood as strings, simple sequences of base pairs. Highthroughput technologies have brought an explosion of population genetics information, including from projects like HapMap [1], the 1000 Genomes Project [3], and UK10K [52]. The question is emerging: how can we use population genetics information to improve accuracy of genomic analyses? This has fueled interest in techniques that depart from a linear string as point of reference for all individuals and toward pan-genome representations $[36,50]$ more inclusive of genetic variation.

While methods for including variants in the reference are growing in number $[18,21,25,43,44,48,49]$, there is little or no work on how to choose which variants to include. Past studies have made such decisions in ad hoc ways, with some filtering according to allele frequency [21, 31], ethnicity [43], or both [49].

Here, we examine the advantages and disadvantages of adding variants to the reference. We show that the disadvantages are important to measure, since simply adding more variation to the reference eventually reduces alignment accuracy. We suggest efficient models for scoring variants according to the effect on accuracy and "blowup" (computational overhead), and further show that these scores can be used to achieve a balance of accuracy and overhead superior to current approaches. For example,

\footnotetext{
*Correspondence: langmea@cs.jhu.edu

${ }^{1}$ Department of Computer Science, Johns Hopkins University, Baltimore, USA

${ }^{2}$ Center for Computational Biology, Johns Hopkins University, Baltimore, USA
}

extrapolating to a whole-human DNA sequencing experiment at 40-fold average coverage, we estimate that a wellengineered augmented reference can yield about $4.8 \mathrm{M}$ more correctly aligned reads and $1.2 \mathrm{M}$ fewer incorrectly aligned compared to the linear reference. Our methods for selecting variants also reduce reference bias, a chief goal of graph genomes. Finally, we compare the accuracy yielded by our methods to that achieved using an ideal personalized graph genome. We show that our methods approach the ideal much more closely than both linear genomes-even when they are modified to contain only major alleles-and graph genomes built on different sets of variants.

These methods are implemented in a new open source software tool called FORGe. We demonstrate FORGe in conjunction with the HISAT2 [25] graph aligner and with another aligner based on the Enhanced Reference Genome [43]. But FORGe's models and methods are suitable for any aligner that can include variants in the reference.

\section{Read alignment with variants}

Read alignment is the process of determining each read's point of origin with respect to a reference genome. The origin can be ambiguous, and reported alignments can be incorrect [29]. Repetitive genomes and sequencing errors contribute to this problem [26, 29]. Importantly, genetic differences between donor and reference genomes also contribute. Alignments overlapping positions where the 
genomes differ-i.e., where the donor genome has a nonreference allele-are systematically penalized. This can (a) reduce the correct alignment's score below the threshold considered significant by the aligner, (b) cause the aligner's heuristics to miss the correct alignment, and (c) cause the correct alignment's score to fall below the score at a different, incorrect location. The problem is magnified in hyper-variable regions such as the major histocompatibility complex (MHC) [12, 17]. It is also problematic when individuals differ dramatically, e.g., if they are from distinct inbred strains [44], or when downstream analyses are vulnerable to allelic bias, such as when detecting allele-specific expression $[4,9,43]$ or calling heterozygous variants $[10,15]$.

Augmenting the reference genome with known variants helps in two major ways. First, it reduces the genetic distance between donor and reference genomes, removing the tendency to penalize correct alignments that overlap non-reference alleles. Second, it avoids the allelic bias, also called "reference bias," [9] that results when one donor haplotype resembles the reference more closely than the other(s).

There are many proposals for how to include and index genetic variants along with the reference genome. Two early approaches were GenomeMapper [44] and the Enhanced Reference Genome [43]. GenomeMapper came from a project to sequence many inbred strains of Arabidopsis thaliana, and it used a graph representation and an accompanying $k$-mer index to represent and align to a graph representing all strains. The Enhanced Reference Genome [43], which specifically addresses reference bias for allele-specific expression, included variants by taking the non-reference allele along with flanking bases and appending these "enhanced segments" to the linear reference genome. Since the resulting reference is linear, a typical read aligner like Bowtie [27] can be used.

Several studies have expanded on these ideas. deBGA [30] uses a colored De Bruijn graph [22] and accompanying hash-table index. BWBBLE [21] and gramtools [31] use an FM Index [16] with an expanded alphabet and modified backward-search algorithm to account for variants. GCSA [49] generalizes the compressed suffix array to index not a single reference but a multiple alignment of several references. HISAT2 [25] combines GCSA with the hierarchical FM Index implemented in HISAT [24]. GCSA2 [48] indexes paths in arbitrary graphs and is implemented in the VG software tool [18] which can align reads to such indexes. MuGI [8] and GraphTyper [15] use $k$-mer-based indexes.

Genome assemblies are also evolving along these lines. The GRCh37 and GRCh38 human assemblies [6, 7] include "alt loci," alternate assemblies of hypervariable regions including $\mathrm{MHC}$. Other studies suggest modifying the linear genome by replacing each non-major allele with its major alternative $[11,23]$. This leverages populationlevel information while keeping a linear representation.

While including variants in the reference incurs a computational cost, the nature and magnitude of the cost depends on the method. For some methods, the most direct manifestation is in the size of the reference genome and index. The Enhanced Reference Genome [43] and its index both grow with the number and length of the "enhanced segments" added to cover all windows containing non-reference alleles. The number of segments required to cover a set of $k$ variants within the span of a single read is $2^{k}-1$. Even if this phenomenon is isolated in a few areas of the genome (e.g., hypervariable regions), the appearance of $k$ in the exponent means it can be very significant. The GCSA method [49] used in HISAT2 [25] incurs similar blowup in its "path doubling" step. We elaborate in Additional file 1: Note S1, Additional file 1: Figure S1, and Additional file 1: Figure S2, including specific discussions of how the cost manifests in different methods, how it leads to more ambiguity in the reference genome which can ultimately lead to reduced accuracy, and how it can be controlled.

\section{Variant selection and evaluation}

Past efforts that evaluated graph aligners have been selective about what variants to include in the graph, but without a clear rationale. Some included all variants from a defined subset of strains or haplotypes [8, 30,44] or from a database such as the 1000 Genomes Project callset [3] or dbSNP [46]. In some cases, variants were filtered according to ethnicity, e.g., keeping just the Finnish 1000 Genomes individuals [49] or the Yoruban HapMap [1] individuals [43]. The ERG study (concerned with allelespecific expression) excluded variants outside annotated genes. The gramtools study [31] used 1000 Genomes variants but excluded those with observed allele frequency less than 5\%. GraphTyper [15] used dbSNP variants in one experiment, excluding single-nucleotide variants (SNVs) with under $1 \%$ frequency in all populations. HISAT2's software for selecting variants to include filters out SNVs with an allele frequency of under $10 \%$ in some cases [25].

Here we explicitly model the variants according to their effects on alignment, and we provide methods for choosing an optimal set based on those models. We apply these methods in combination with two different augmentedreference alignment methods and compare to a range of relevant competing methods, including a linear reference with reference alleles, a linear reference with all-major alleles, and an ideal "personalized" reference that customized to fit the donor individual's alleles (including at heterozygous positions) as closely as possible. This experimental design allows us to make statements about how our methods affect accuracy, how those effects vary with genomic region, how close the methods come to achieving 
ideal accuracy, and how practical current graph alignment methods are overall.

\section{Results}

\section{Strategy}

FORGe works in cooperation with a variant-aware read aligner (graph aligner) such as HISAT2 [25]. Consider the alignment process as being divided into offline (index building) and online (alignment) stages. FORGe operates in the offline stage. Specifically, FORGe takes a reference genome (FASTA format) and catalog of variants and their frequencies in the population (Variant Call Format). FORGe can also use phasing information when provided in the VCF. FORGe then uses a mathematical model to score each variant according to its expected positive and negative impacts on alignment accuracy and computational overhead. The model could consider factors such as the variant's frequency in a population, its proximity to other variants, and how its inclusion affects the repetitiveness of the graph genome. Using these scorestogether with a parameter for the overall percentage or number of variants to include-FORGe outputs the topscoring subset of variants, which can then be fed to the index-building component of a graph alignment tool like HISAT2's hisat2-build program. In the online stage, the aligner uses this FORGe-customized index to align the sequencing reads.

\section{Simulation}

We used Mason 0.1.2 [20] to simulate reads (details in Additional file 1: Note S2). Mason simulates sequencing errors and base quality values. Mason also annotates each read with information about its true point of origin. We disabled Mason's facility for adding genetic variants, since we simulate from already-individualized references. We classify an alignment as correct if its aligned position in the reference is within $10 \mathrm{nt}$ of the true point of origin. If the aligner reports several alignments for a read, we consider only the primary alignment-of which there is exactly one per aligned read, usually with alignment score equal to or greater than all the others-when determining correctness.

\section{Alignment}

We tested FORGe with two read alignment strategies capable of including variants in the reference: HISAT2 [25] and the Enhanced Reference Genome (ERG) [43]. HISAT2 is a practical graph aligner that we hypothesized would benefit from careful selection of genetic variants to include. The ERG is simple and compatible with linear aligners like Bowtie. We use ERG only with short unpaired reads $(25 \mathrm{nt})$ to test the hypothesis that the seed-finding step of an aligner can benefit from including FORGe-selected variants. Adapting the ERG approach to paired-end alignment is probably not practical (see the "Discussion" section).

In its offline stage, HISAT2 takes a linear reference genome and a VCF file with single-nucleotide variants and indels. HISAT2 uses GCSA indexing [49] to build a graph-genome index. The resulting graph is the generating graph for all combinations of reference (REF) and included alternate (ALT) alleles. HISAT2 also provides software that, starting from a VCF file (or the UCSC "Common SNPs" track, derived from dbSNP [46]), selects a subset of variants to include. It filters in two ways. First, it excludes variants with allele frequency under $10 \%$. Second, where variants are densely packed, it imposes artificial haplotype constraints to avoid the exponential blowup that results from considering all combinations of REF and ALT alleles. We call this the HISAT2 auto method.

We also tested FORGe with our implementation of the ERG [43]. ERG's offline phase starts with a linear reference genome and a variant file. It builds an augmented reference genome by adding enhanced segments: reference substrings that include ALTS and flanking context. The amount of context depends on a user-specified window size, $r$, which typically equals the maximum read length. When $n$ variants co-occur in a window, $2^{n}-1$ enhanced segments are added to cover all combinations of ALT and REF alleles. The original ERG study limited growth by considering only the leftmost $k$ variants per length- $r$ window, with $k=5$ in practice. We use a variation on this limit: if a window contains more than $k$ variants, we consider (a) the leftmost variant, and (b) the $k-1$ other variants with highest allele frequency according to the input VCF. Including the leftmost guarantees that each variant has its ALT included in at least one of the overlapping enhanced segments. We also set the limit higher $(k=15)$ by default. While $k$ is configurable, we used the default in all experiments here. After adding enhanced segments to the reference, we indexed it with Bowtie [27]. In the online stage, we used Bowtie to align to the enhanced reference. Details on our ERG implementation are in Additional file 1: Note S3.

In all experiments, we ran HISAT2 with the $-\mathrm{k}$ 10,--no-spliced-alignment, and --no-tempsplicesite options. In the ERG experiments, we ran Bowtie with the $-\mathrm{v} 1$ option to allow alignments with up to one mismatch. Note that HISAT2 is able to find alignments with mismatches, insertions, or deletions, whereas Bowtie can only find alignments with mismatches. In all cases, we used Python's rusage module to measure peak resident memory usage and we used the Linux time utility to measure real ("wall clock") running times. All tools were run using a single thread. 


\section{Variant models}

As detailed in the "Methods" section, FORGe has two main models for ranking and selecting variants to include in the reference. First is Population Coverage (Pop Cov), which scores variants according to allele frequency. Second is Hybrid, which weighs both a variant's allele frequency and the degree to which its addition would make the reference more repetitive. Additionally, we evaluated versions of these two models enhanced with a blowup avoidance strategy that, at variant adding time, dynamically down-weights candidates that are close to alreadyadded variants. These versions are called Pop Cov+ and Hybrid+. All of these strategies are detailed in the "Methods" section.

\section{Chromosome 9 simulation}

We tested FORGe in a series of simulation experiments. We used human chromosome 9 from the GRCh37 assembly [6]. GRCh37 was chosen to match the coordinates for the official 1000 Genomes Project Phase-3 variants [3]. We simulated sequencing reads from chromosome 9 of NA12878, a female from the CEPH (Utah residents with Northern and Western European ancestry) group studied in the 1000 Genomes Project. Specifically, we generated 10 million unpaired Illumina-like reads from each haplotype of NA12878 for a total of 20 million reads. Each read comes from one of the two haplotypes. We created a VCF file containing all single-nucleotide variants (SNVs) appearing in chromosome 9 in at least one 1000-Genomes individual, excluding NA12878 and family members. The resulting file contained 3.4 million SNVs. Details on how this set of SNVs was obtained are presented in Additional file 1: Note S4. We used the Pop Cov, Hybrid, Pop Cov+, and Hybrid+models to score the 3.4 M SNVs. The Hybrid and Hybrid+ models used phasing information, whereas the Pop Cov and Pop Cov+ models did not (explained in Methods). We compiled subsets of SNVs consisting of the top-scoring $0 \%, 2 \%, 4 \%, 6 \%, 8 \%, 10 \%, 15 \%$, and $20 \%$ up to $100 \%$ in 10 point increments.

HISAT2 Figure 1 shows alignment rate and accuracy when using HISAT2 to align our simulated 100 nt reads to the genome indexes created with hisat2-build. The leftmost point (or in the case of Fig. 1c, the point labeled $0 \%$ ) corresponds to a HISAT2 index with no SNVs added, i.e., a linear reference genome. The diamond labeled Major Allele corresponds to a linear reference with all major alleles; i.e., with every SNV set to the allele that was most most frequent among CEU individuals in the filtered callset. The diamond labeled HISAT2 auto corresponds to the pruned set obtained by running HISAT2's scripts. The diamond-labeled Personalized shows results when aligning to a personalized NA12878 genome with all nonreference homozygous (HOM) alleles replaced by their
ALT versions and all heterozygous (HET) SNVs added as variants, so that neither REF nor ALT are penalized at alignment time. This is not a realistic scenario, but helpful for assessing how close the tested methods come to the personalized-genome ideal. Plotted lines show results obtained when adding progressively larger subsets of SNVs to the graph genome, prioritized by model score.

Figure 1a, b shows alignment rate and fraction of alignments that are correct (henceforth "correctness") as a function of the number of SNVs included in the genome. For all models except Hybrid+, peak alignment rate and correctness occur in the 8-12\% range of SNVs included. All the FORGe models at their peak achieve higher alignment rate and correctness than the major-allele and HISAT2 methods. When greater fractions of variants are included-more than around $12 \%$-alignment rate and correctness generally decrease. Correctness eventually decreases to a level only somewhat higher than that achieved by the linear reference, showing that alignment suffers when too many variants are included. Figure 1d, e is similar to $\mathrm{a}$ and $\mathrm{b}$ but show alignment rate and correctness as a function of HISAT2's memory footprint at alignment time. While FORGe's models at their peak have a roughly $50 \%$ larger memory footprint than the linear references (both major-allele and reference-allele), they use roughly half the memory of the "HISAT2 auto" method.

Figure 1c plots a point or a parametric curve for each indexing strategy and model. The vertical axis is the fraction of reads (not alignments) that aligned correctly, and the horizontal axis is the fraction of reads that aligned incorrectly. Notable points on the curves are labeled with the fraction of SNVs included. Diamonds mark points on the curves with maximal $y-x$, where $y$ is fraction correct and $x$ is fraction incorrect. This is a combined measure for alignment rate and accuracy, and maximal values are reached in the $8-10 \%$ range of SNVs included (except Hybrid+, which peaked at 30\%). The best performing are superior to (above and to the left of) the linear-genome methods, the "HISAT2 auto" method, and to the genome obtained by adding all of the SNVs (labeled 100\%). The best-performing graph genomes come much closer to the personalized-genome ideal than the other methods.

It is notable that the alignment rate curves in Fig. 1a, $\mathrm{b}, \mathrm{d}$ and e eventually trend downward. Like most read aligners, HISAT2 uses heuristics to limit the effort spent aligning reads to many repetitive regions of the same reference genome. HISAT2 is unusual in that when a read has too many repetitive alignments, it will abort and leave the read unaligned. Bowtie does not have this heuristic; rather, Bowtie chooses one best-scoring alignment to report even when the read has many repetitive alignments. Because of this, HISAT2's alignment rate decreases as more variants are included and the genome becomes more repetitive. 

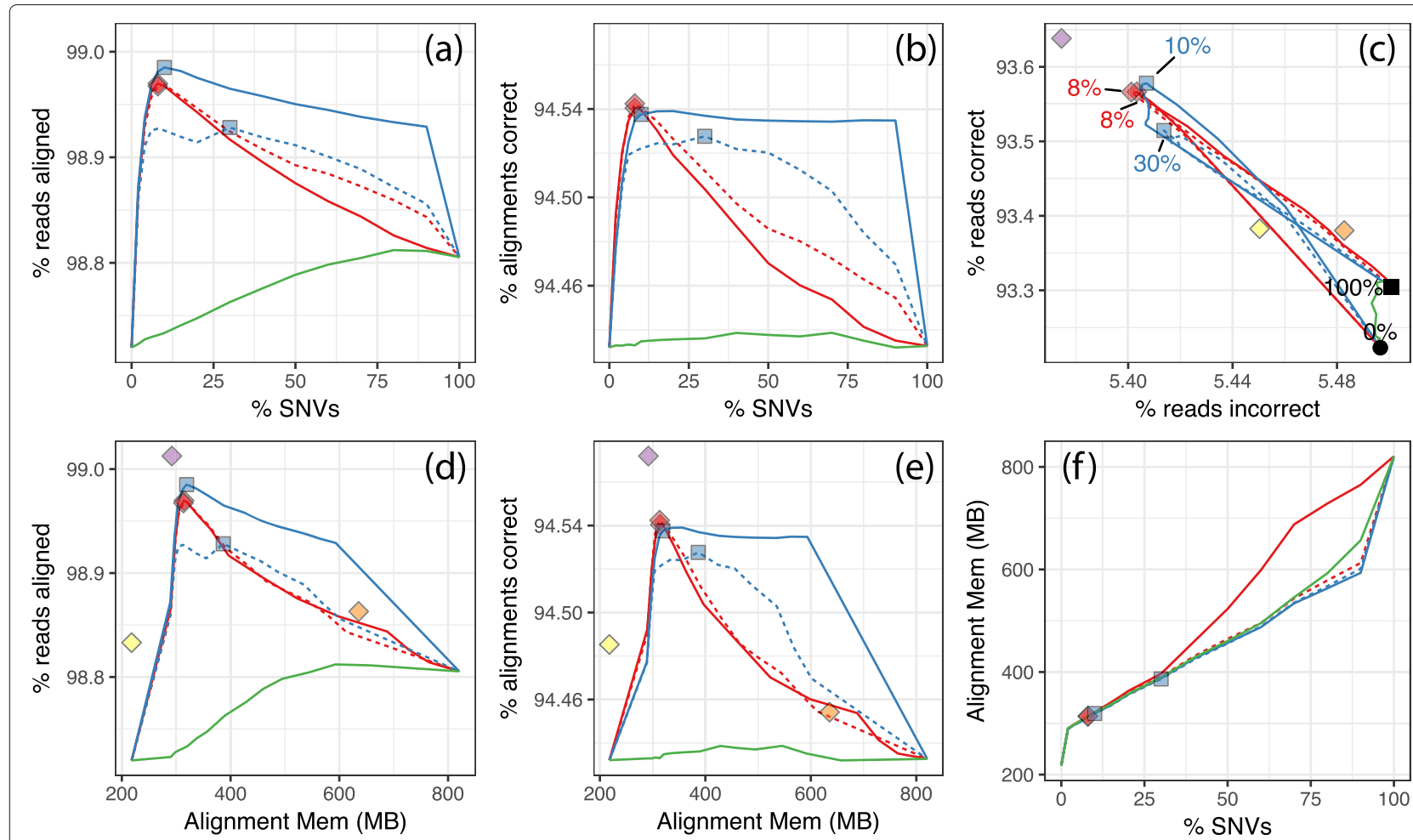

$$
\begin{array}{ll}
\diamond \text { Population coverage } & \square \text { Hybrid } \diamond \text { Random } \\
\diamond \text { Major Allele } & \diamond \text { HISAT2 Auto } \diamond \text { Personalized }
\end{array}
$$

- Standard $\quad \cdots$... Blowup avoidance (+)

Fig. 1 Results from NA12878 simulation for GRCh37 Chromosome 9. 100 nt unpaired reads were simulated from Chromosome 9 with NA12878's variants included. FORGe and HISAT2 created and indexed augmented reference genomes with various variant sets. Besides the Pop Cov and Hybrid rankings, we also included a strategy that gave variants random ranks ("Random"). $\mathbf{a}$ and $\mathbf{d}$ show the fraction of reads aligned. $\mathbf{b}$ and $\mathbf{e}$ show the fraction that aligned correctly to the simulated point of origin. c plots a parametric curve of the fraction of reads with a correct alignment (vertical) versus the fraction with an incorrect alignment (horizontal). Lines follow measurements made over a range of fractions of SNVs, with points for $0 \%$, $2 \%, 4 \%, 6 \%, 8 \%, 10 \%, 15 \%$, and $20 \%$ up to $100 \%$ in 10 point increments. The diamond labeled HISAT2 auto is an augmented genome produced using HISAT2's pruning scripts. The diamond labeled Major allele ref is a linear reference with all positions set to the most frequent allele. Other diamonds indicate the SNV fraction maximizing $y-x$, where $y$ is the fraction of reads aligned correctly and $x$ is the fraction aligned incorrectly. The HISAT2 and Major allele diamonds are excluded from panels $\mathbf{a}, \mathbf{b}$, and $\mathbf{f}$ because there is no clear way to measure the fraction of variants included by these methods. The black filled circle and square in panel c represent measurements when $0 \%$ and $100 \%$ of variants are included, respectively

A known drawback of graph aligners is that accuracy and overhead can suffer when many variants co-occur in a small window of the genome. To measure the impact this has on FORGe's models, we also plotted results using blowup avoiding versions of the Pop Cov and Hybrid models (Fig. 1, dotted lines), called Pop Cov+ and Hybrid+. These versions will, when selecting variants to add, deprioritize variants that are near already-added variants. We observed that blowup avoidance had a minimal impact on the shape of the Pop Cov curve; e.g., Fig. 1d, e shows the solid and dotted lines for Pop Cov on top of each other. Notably, blowup avoidance did cause the alignment memory to increase more slowly with respect to the number of added variants for the Pop Cov ranking (Fig. 1f). For the Hybrid model, blowup avoidance did not change the relationship between memory footprint and number of variants added (Fig. 1f) and had an adverse effect on alignment rate and correctness. This is likely because the Hybrid model already takes clustered variants into account in its $k$-mer counts.

We repeated these experiments for paired-end reads (Additional file 1: Figure S3) and the results closely followed those in Fig. 1. Alignment rate and accuracy both increased when using paired-end reads, since an accurate alignment for one end can "rescue" the other in the presence of ambiguity. Peak accuracy (maximal $y-x$ ) was achieved at the same SNV fraction except in the case of the Hybrid ranking, which peaked at $15 \%$ rather than at $10 \%$.

We also repeated these experiments for reads simulated from Yoruban (YRI) individual NA19238, also sequenced in the 1000 Genomes Project (Additional file 1: Figure S4). As we did for NA12878, we excluded variant calls for NA19238 and family members before providing variants 
to the model for scoring. These results also closely followed those in Fig. 1, with accuracy and recall peaking at a somewhat higher percentage of variants included $(15 \%$ for YRI compared to 8-10\% for CEU), likely due to YRI's greater divergence from the reference. We return to this in the "Discussion" section.

Finally, we repeated the unpaired NA12878 experiment including both SNVs and indels in the FORGe analysis (Additional file 1: Figure S5). Whereas previous experiments modeled and scored 3.4 M SNVs, here we modeled and scored 3.4 M SNVs and 131k indels, composed of 49k insertions ranging in length up to $411 \mathrm{nt}$ and $82 \mathrm{k}$ deletions up to $92 \mathrm{nt}$. Given these variant scores, we selected top-scoring fractions, built indexes, simulated reads from NA12878 (both SNVs and indels included) and performed alignments as before. When assessing correctness of the resulting read alignments, we took coordinate shifts due to indels into account. Overall the results are similar to those in Fig. 1. While there is a slight drop in peak alignment and correctness rate, the rates varied over a wider range of percentages relative to the SNV-only experiment. Maximal $y-x$ occurred at slightly higher variant fractions relative to the SNV-only experiment: $10 \%$ for Pop Cov and Pop Cov+, 15\% for Hybrid and 30\% for Hybrid+.
Enhanced Reference Genome Figure 2 shows alignment rate and correctness when using Bowtie [27] to align simulated $25 \mathrm{nt}$ reads to enhanced references constructed with the ERG method [43]. We used shorter reads and configured Bowtie to find alignments with up to 1 mismatch $\left(\begin{array}{ll}-\mathrm{v} & 1\end{array}\right)$ to mimic the seed alignment step of seed-andextend aligners.

Unlike HISAT2, Bowtie always reports an alignment if one is found, regardless of how repetitively the read aligns. Consequently, the alignment rate shown in Fig. 2a and d strictly increases as variants are added to the graph. Apart from that, the results reinforce those from Fig. 1. Peak correctness occurs at a relatively small fraction of SNVs (6-20\%). As more variants are added, correctness eventually decreases, though the Hybrid ranking does not suffer this drop until over 70\% of SNVs are included. The alignment-time memory footprint of the best-performing FORGe indexes is higher than that of the linear reference; e.g., including the top 6\% of Pop Cov+-scored SNVs increases the footprint 29\%, from 127.9 MB to $165.0 \mathrm{MB}$. But it is a fraction of the size of the index when $100 \%$ of variants are included (1.87 GB). Blowup avoidance (Fig. 2, dotted lines) had a somewhat minor effect on alignment rate and correctness for Pop Cov, and a clear negative
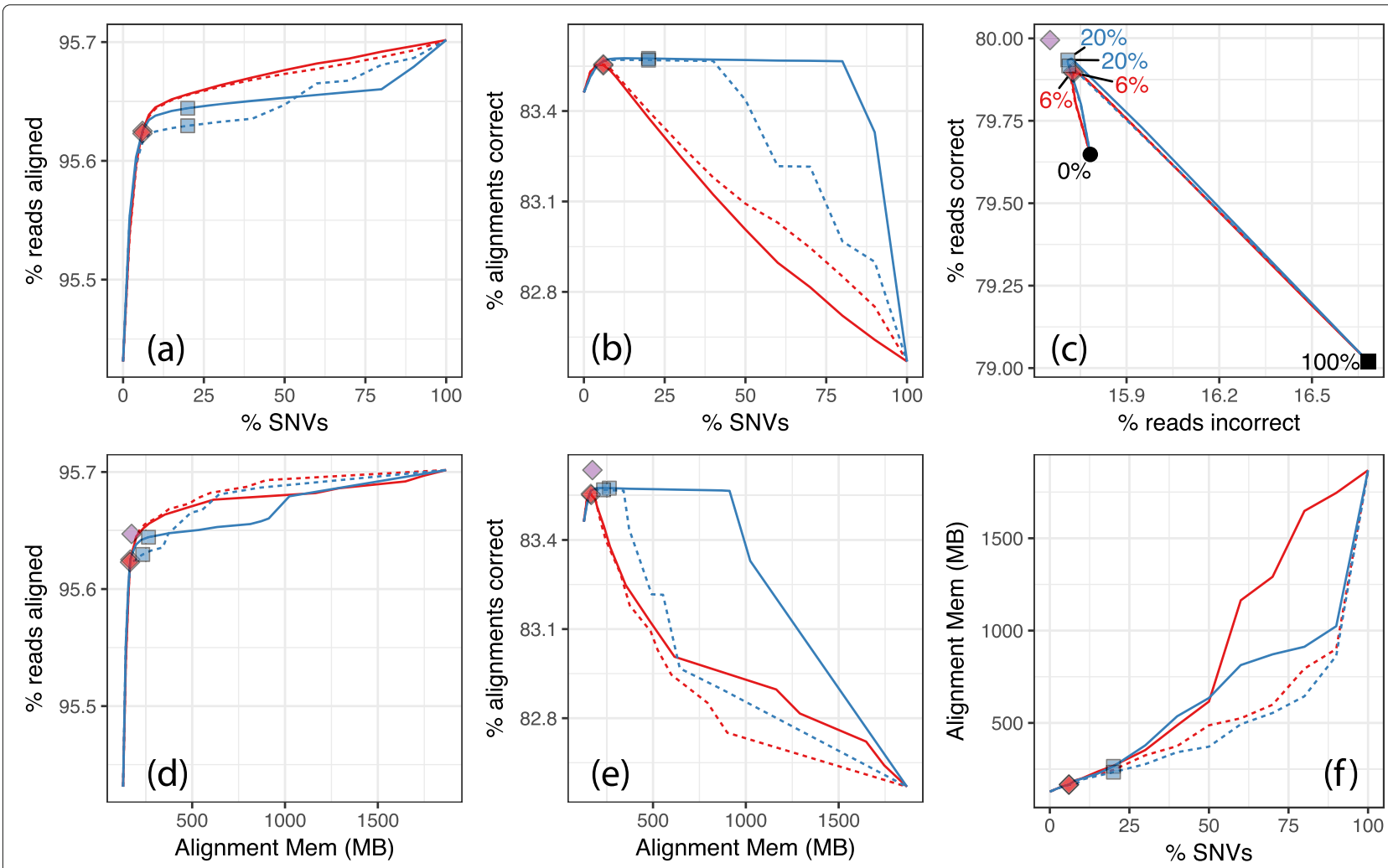

$\diamond$ Population coverage $\square$ Hybrid $\diamond$ Personalized

- Standard -... Blowup avoid

Fig. 2 Results from aligning simulated 25 nt unpaired reads to GRCh37 chromosome 9 using the ERG+Bowtie approach. Panels and plots have the same meaning as for Fig. 1 except that HISAT2 auto and Major allele ref diamonds are omitted here 
effect for Hybrid. On the other hand, it slowed the rate of index growth for both models at low and intermediate fractions of SNVs (Fig. 2f).

\section{Stratification by variant density, variant rarity, and repetitiveness}

Figure 1c showed that when we move from 0 to $8 \%$ of variants included in the augmented reference, the number of correct alignments increases by about 0.4 percentage points (as a fraction of reads) and the number of incorrect decreases by about 0.1 points. Though these may seem like small differences, in a study with 1.2 billion reads-approximately the number of unpaired 100 nt unpaired reads required to cover the human genome to 40 -fold average depth-this would yield about $4.8 \mathrm{M}$ more correctly aligned reads and 1.2 M fewer incorrectly aligned.

Still, we hypothesized that certain read subsets might be affected more dramatically by the inclusion of variants. To this end, we measured alignment rate and correctness when we varied the number of alternate alleles overlapped by a read (Fig. 3a-c), whether the alternate allele was common or rare (Fig. $3 \mathrm{~d}-\mathrm{f}$ ) and what kind of genomic region or repeat the read originated from Fig. $3 g-i$. The measurements studied here are the same as those presented in Fig. 1, but filtered as described below.

Figure $3 \mathrm{a}-\mathrm{c}$ shows alignment rate and correctness stratified by the number of non-reference SNVs overlapped by a read. To obtain these subsets, we first removed reads originating from reference-genome regions deemed
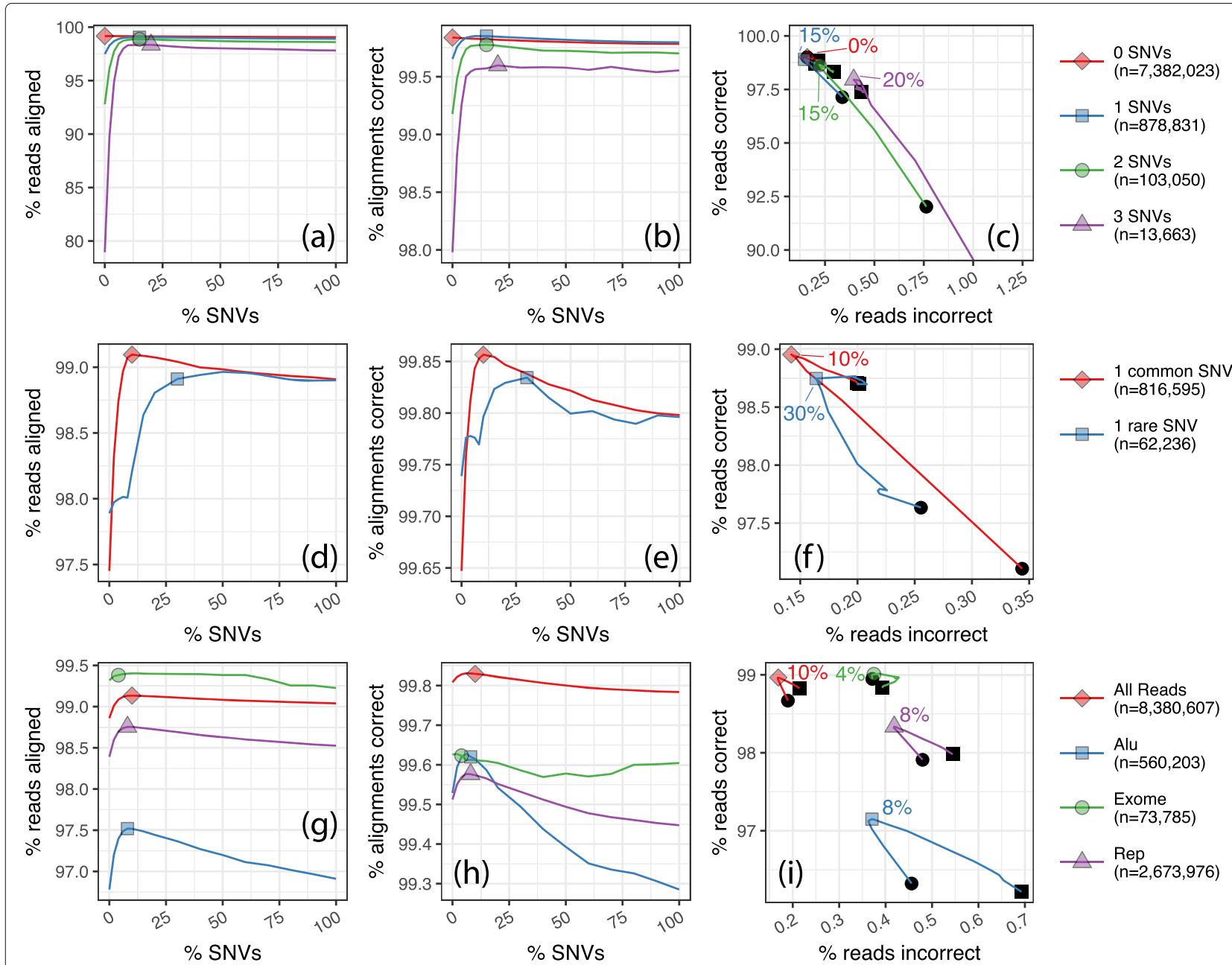

Fig. 3 First row: Results for simulated reads stratified by the number of SNV alternate alternate alleles overlapped by the read. Reads overlapping regions with high DangerTrack [13] score_-indicating the regions are difficult to align to_-are omitted. Second row: Results for simulated reads overlapping exactly one common alternate allele (and no other alternate alleles) and reads overlapping exactly one rare allele. Reads overlapping regions with high DangerTrack [13] score, indicating the regions are difficult to align to. Third row: Results for simulated reads stratified by region of origin. Regions examined are regions labeled with the "Alu" family by RepeatMasker, regions captured by the Nextera exome sequencing protocol ("Exome"), and regions labeled with any repeat family by RepeatMasker ("Rep") 
repetitive by DangerTrack [13] (score over 250). We did this after finding that these regions had a combination of low SNV density and repetitive content that caused the 0 -SNV stratum to behave very differently from the others. Reads containing 1 or more SNVs undergo a rapid increase in alignment rate and correctness from 0 to $10 \%$ of SNVs. Beyond 10\%, all strata experience a slow decrease in alignment rate and correctness up to $100 \%$ of SNVs added. The 0-SNV stratum has decreasing alignment rate and correctness across the whole range, as expected since the addition of variants cannot help (since the reads lack alternate alleles) but can harm alignment by increasing the repetitiveness of the reference. Strata with more SNVs experience a more dramatic rising-and-falling pattern; for the 3-SNV stratum, alignment rate varies from about $80-98 \%$. While curves for the various strata have different shapes, all peak at a relatively low SNV fraction: $20 \%$ or lower.

Figure $3 \mathrm{~d}-\mathrm{f}$ show alignment rate and correctness for reads containing a single rare SNV allele (1000 Genomes frequency $<0.5$ ) versus reads containing a single common SNV allele ( $\geq 0.5$ ). In both cases, we considered only reads with a single non-reference allele. Rare-SNV reads peak lower and at a higher SNV fraction than common-SNV reads for both alignment rate and correctness (Fig. 3d-f). This is expected, since the Pop cov model prioritizes common over rare SNVs. In other words, by the time a rare variant is added, many common variants have already been added, making the genome more repetitive.

Figure $3 \mathrm{~h}-\mathrm{j}$ shows alignment rate and correctness for reads stratified by feature of origin. We analyzed reads originating from (a) RepeatMasker-annotated repetitive regions (http://www.repeatmasker.org), (b) RepeatMasker-annotated "Alu" repeats, (c) regions captured by the Nextera exome sequencing protocol, and (d) all reads.

Reads from repetitive regions generally had lower alignment rate and correctness compared to all reads. As before, alignment rate and correctness curves peaked at low SNV fractions: $10 \%$ or lower. Reads from more repetitive features were more sensitive to the number of variants included in the reference, as evidenced by the vertical spans of the curves.

In a related experiment, we examined the graph genome's effect specifically on the hypervariable MHC region. We simulated reads from NA12878 Chromosome 6 and used HISAT2 to align to both a linear and a graph genome augmented with the top-scoring $10 \%$ of SNVs. We visualized the read-alignment pileup in the hypervariable MHC region using IGV [51] (Additional file 1: Figure S6). Qualitatively, the pileup for the augmented reference looks superior-with more coverage in variantdense regions and with more even overall coverage-to the pileup for the linear reference.

\section{Ethnicity specificity}

We also studied how ethnicity-specific augmented references, advocated in other studies [2,33, 47], can improve alignment. We used FORGe to select variants from two lists: one with variants drawn from and scored with respect to the overall 1000-Genomes phase-3 callset, and another drawn from and scored for just the CEU individuals. In both cases, variants private to NA12878 and family members were excluded and reads were simulated from NA12878.

Figure 4 shows alignment rate and correctness when aligning to CEU-specific and pan-ethnic references. As expected, the CEU-specific reference yielded higher alignment rate and correctness. CEU-specific curves also peaked at lower numbers of SNVs compared to panethnic. However, the differences were only a few hundredths of a percentage point and cover only a small fraction of the remaining distance to the ideal point. Looking at this another way, if we extrapolate the results to a whole-genome DNA sequencing experiment with 40fold average coverage, around 250,000 alignments would be affected. We return to these small differences in the "Discussion" section.

\section{Whole human genome}

Simulated reads To show our methods generalize to whole genomes, we repeated experiments like those presented in Fig. 1 using the full GRCh37 reference. We gathered 80.0 million SNVs from the Phase-3 callset of the 1000 Genomes Project [3]. We used FORGe's Pop Cov+ model to score SNVs and compiled subsets consisting of the top-scoring $2 \%, 4 \%, 6 \%, 8 \%, 10 \%, 15 \%$, and $20 \%$ up to $100 \%$ in 10 point increments. We built graph-genome indexes for each using HISAT2.

We used the Pop Cov+ model because the others required excessive time and/or memory; specifically, the Pop Cov model (without blowup avoidance) produced a set of variants that HISAT2 was unable to index in a practical time and space budget (Additional file 1: Note S5) and the Hybrid and Hybrid+ models required excessive time for the step that generates the FASTA file for $G *$ due to exponential blowup (Additional file 1: Note S6).

Figure $5 \mathrm{a}, \mathrm{b}$ plots HISAT2 alignment rate and correctness as a function of the SNV fraction. We aligned 20 million 100 nt unpaired reads from simulated from NA12878. We omitted NA12878 and family members from variant selection. Results using the ideal personalized index are also shown for comparison. Maximal $y-x$, where $y$ is the fraction of reads aligned correctly and $x$ is the fraction aligned incorrectly, occurred at $10 \%$ of SNVs (Fig. 5c). Interestingly, the maximal point does not approach the personalized-genome ideal point as closely here as it did for the chromosome-9 experiment (Fig. 1). This seems to be due to the added ambiguity that comes when variants 

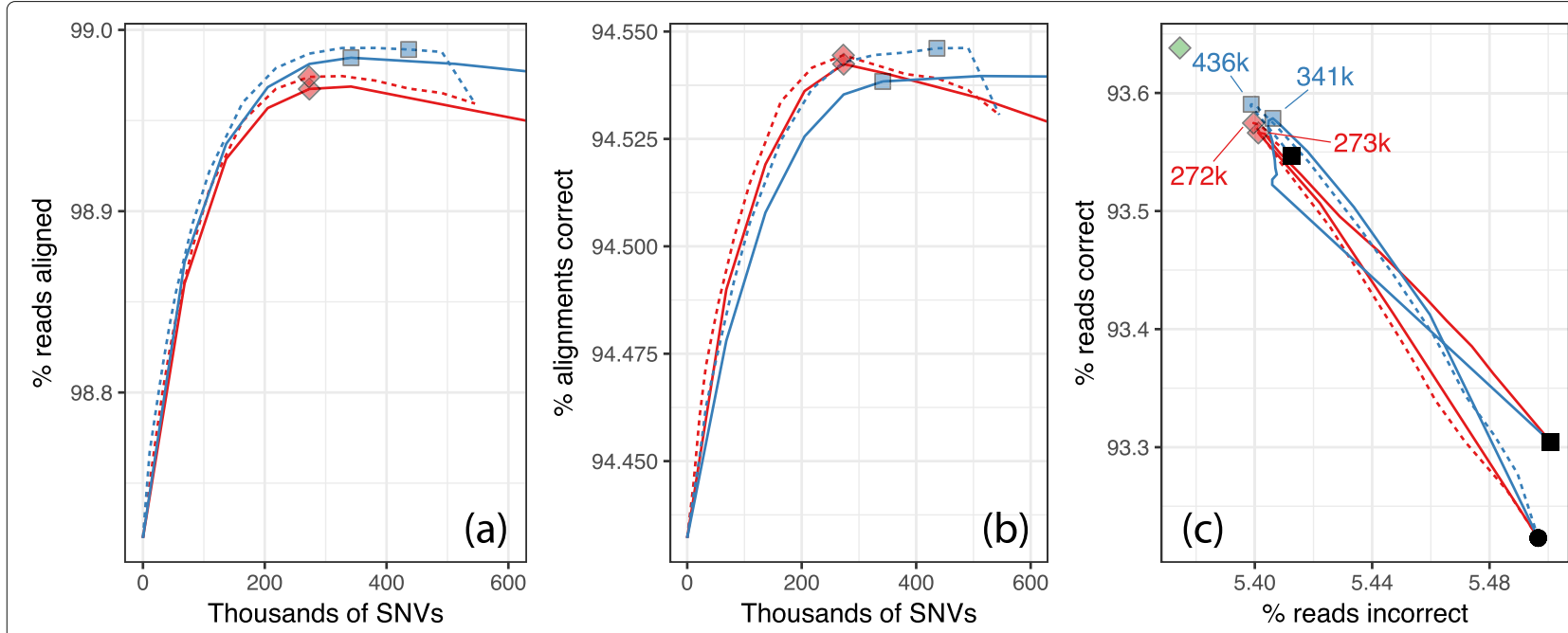

— All -... CEU only

Population coverage $\square$ Hybrid $\diamond$ Personalized

Fig. 4 Results from chromosome-9 NA12878 simulation when using an ethnicity-specific ("CEU") versus a pan-ethnic ("All") augmented reference. Reads are $100 \mathrm{nt}$ and unpaired and the plots have similar axes as the plots in Fig. 1 panels $\mathbf{a}-\mathbf{c}$. Since we are assessing two ethnicities, each with a different total number of variants, the horizontal axes in panels $\mathbf{a}$ and $\mathbf{b}$ and the peak points in panel (c) are labeled with absolute number of variants included rather than percentages

in all non-chromosome-9 portions of the genome are added (Additional file 1: Figure S7).

Platinum reads, SNVs We conducted further experiments using a set of 1.57 billion real 100 nt unpaired sequencing reads from the Platinum Genomes Project [14] (accession: ERR194147). Like the simulated reads, these also come from NA12878. We gathered a set of 80.0 million SNVs from the 1000 Genomes phase-3 callset, omitting variants private to NA12878 and family members. We again used the Pop Cov+ model to select variants.

We cannot assess correctness since the reads were not simulated. Following a prior study [34], we measured the number of reads that align uniquely-where HISAT2 reported exactly one alignment-versus the number that aligned perfectly, matching the reference exactly with no differences. The goal was to capture the variantinclusion trade-off; we hypothesized that adding more

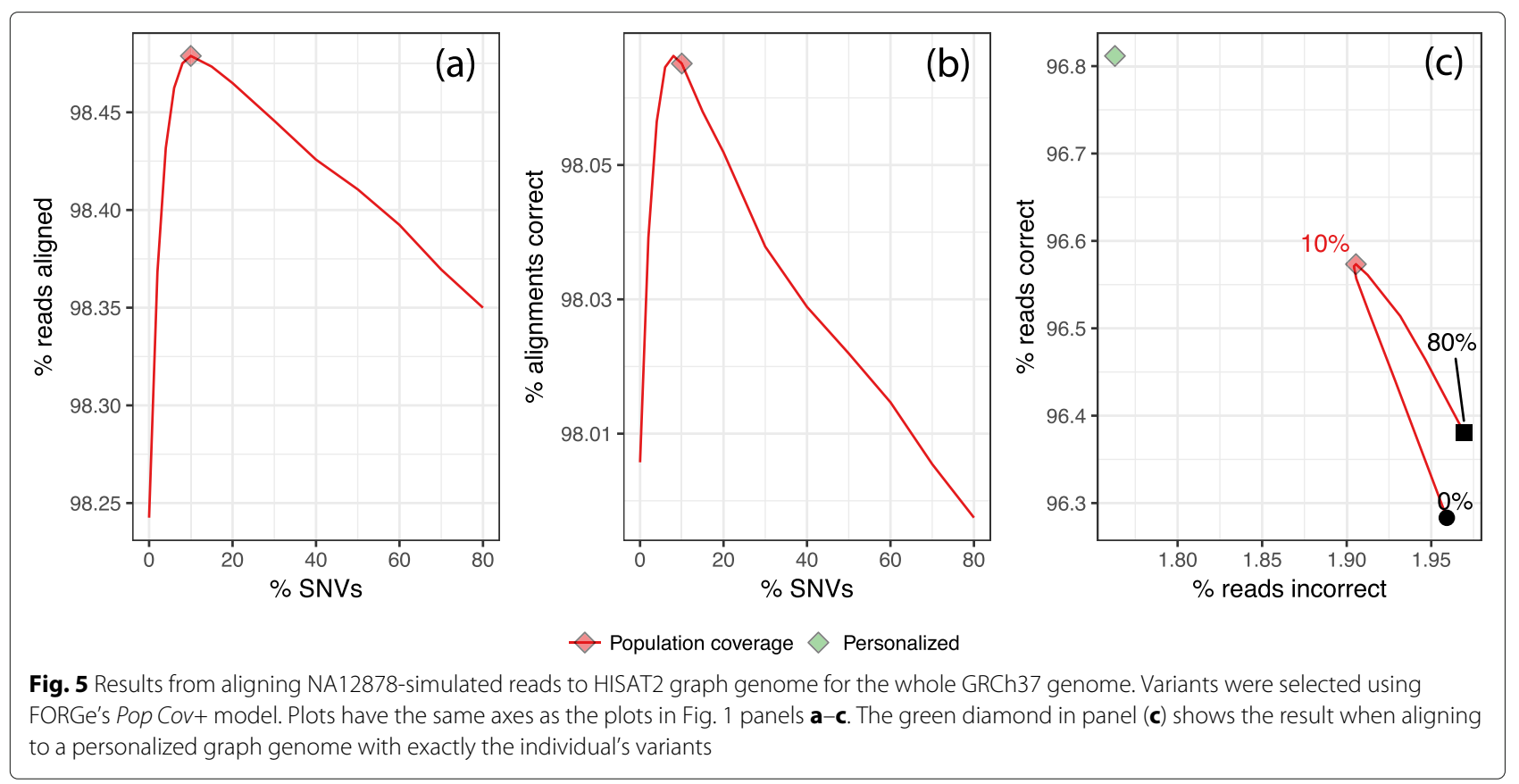


variants will remove the alignment-score penalty associated with known genetic variants (increasing the number of perfect matches) without increasing reference ambiguity (decreasing the number of unique alignments). As shown in Fig. 6a, the points that achieved the peak number of unique plus perfect alignments corresponded to $30 \%$ of the SNVs. This fraction is higher than most of our simulated results, perhaps due to the fact that uniqueplus-perfect is an imperfect proxy for correct-minusincorrect (Additional file 1: Figure S8).

Platinum reads, SNVs, and indels To highlight the effect of including indels in the reference, we repeated the previous experiment but using both SNVs and indels from the 1000 Genomes phase-3 callset. Specifically, we gathered 83.1 million variants, both SNVs and indels, but omitting variants private to NA12878 and family members. We again used the Pop Cov+ model to select variants. We again plotted the number of reads that aligned uniquely versus the number that aligned perfectly (Fig. 6a). The graph genome built from both SNVs and indels achieved peak unique+perfect at $30 \%$ of variants, like the graph built from SNVs alone. However, at every percentage it yields more unique and perfect alignments.

Reference bias We measured how reference bias varies with the fraction of variants included. We analyzed the alignments of the ERR194147 reads to the whole human genome with both SNVs and indels included in in reference. Figure $6 \mathrm{~b}$ shows a series of boxplots summarizing bias at a set of 2.07 million HET SNVs called in NA12878 by the Platinum Genomes Project [14]. The set of 2.07M HETs was chosen by taking all HETs covered by at least 25 reads in all of our experiments. Each boxplot summarizes the fraction of REF alleles $(R E F /(R E F+A L T))$ at the HET site for all 2.07M HETs. As expected, bias decreased as more variants were included. The decrease plateaued at $10-20 \%$ of variants. Beyond $20 \%$, including more variants did further reduce bias, but only slightly. From 20 to $70 \%$ of variants the mean decreased by only 0.00011 .

This is consistent with previous results showing that most of the benefit is achieved at a small fraction of variants.

HLA typing accuracy Finally, to measure FORGe's effect on downstream results, we measured how HLA typing recall vary with the fraction of variants included in the reference. We used the same NA12878/ERR194147 alignments evaluated in previous sections, extracted alignments in the $\mathrm{MHC}$ region, then provided those alignments to the Kourami [28] HLA typing tool to make HLA calls. We repeated this with indexes for all the same variantinclusion fractions evaluated previously. More details on the HLA typing methodology are described in Additional file 1: Note S7. In comparison with linear genome, HLA typing recall and accuracy increased substantially when the highest-scoring $10 \%$ of SNVs were included in the augmented reference. Recall and average coverage plateaued at larger SNV fractions (Additional file 1: Figure S9). Overall, we see that-as we observed in other results-HLA
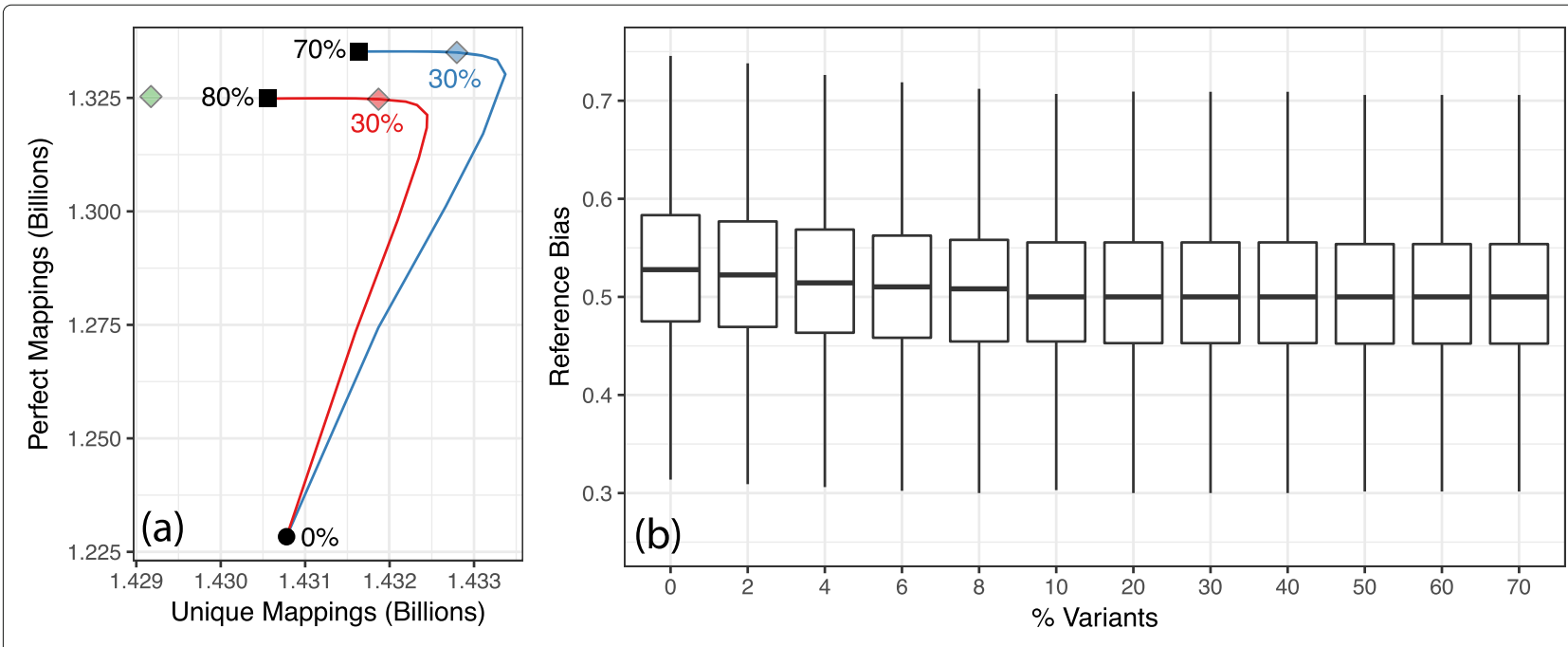

$\diamond$ HISAT2 Auto $\diamond$ SNVs + Indels $\diamond$ SNVs Only

Fig. 6 a Perfect/unique alignment results when aligning real reads. The blue curve is parametric, as a function of the fraction of variants included from $0 \%$ (bottom left) to $80 \%$ (top). The green diamond marks the number perfect and unique mappings for HISAT2's custom variant pruning script applied to the set of SNVs and Indels. Graph genomes were built for SNVs alone (red) and for SNVs and Indels (blue), both ranked with the Pop Cov+ strategy. Blue and red diamonds mark the fractions that achieved the highest sum of unique and perfect alignments. $\mathbf{b}$ Allelic bias for the $2.07 \mathrm{M}$ heterozygous SNVs that met a minimum coverage threshold of 25 in all experiments. Whiskers show the 5th-to-95th percentile range 
allele recall benefits from the addition of a carefullychosen fraction of variants, and that a fraction of only $10 \%$ is sufficient to achieve peak recall.

\section{Methods}

FORGe works in cooperation with a variant-aware read aligner such as HISAT2 [25] or the ERG [43]. The strategy has two stages. In the offline stage, FORGe selects variants to include in the augmented reference based on a variant model - which predicts the pros and cons of including a variant - and a variant limit. The model and limit together constitute a variant inclusion strategy (VIS) that aims for a balance between accuracy and overhead. Once variants have been selected, the aligner software is used to create an index of the augmented reference. The second stage is an online stage where the read aligner aligns reads to the augmented reference using the index.

\section{Offline stage}

Inputs to the offline stage consist of (a) a reference genome, (b) variants in VCF format, (c) a VIS, and (d) a window size $s$. The variant inclusion strategy (VIS) consists of a variant model and a limit on the number or fraction of variants to include. The VIS is the user's most direct means for balancing blowup and alignment accuracy in the augmented reference. We now propose multiple variant models, each aiming to give higher scores to variants that will impart a greater net benefit when considering accuracy and blowup. The window size $s$ is used in three separate places in the software (described below) and should typically be set to the maximum read length.

\section{Variant models}

Let $G_{r e f}$ denote the linear reference genome and $G^{*}$ the complete augmented genome including all variants in the population. Let $G$ be a possible result of a VIS, i.e., an augmented genome that includes a subset of population variants. For simplicity, assume all variants are SNVs (substitutions). Let a localized $s-m e r\langle s, l\rangle$ be a string of length $s$ (the configurable windows size) that matches some combination of alleles in an augmented genome $G$ starting at offset $l$; we also call these simply $\langle s, l\rangle$-mers. For instance, if $G$ is GATYACA, where Y can be either $\mathrm{C}$ or $\mathrm{T}$, then $\langle\mathrm{GAT}, 0\rangle$, $\langle\mathrm{TCA}, 2\rangle$, and $\langle\mathrm{TTA}, 2\rangle$ are all $\langle 3, l\rangle$-mers of $G$.

For an $\langle s, l\rangle$-mer $\sigma$, let $p(\sigma)$ be the probability a random $\langle s, l\rangle$-mer drawn from a random individual in the population equals $\sigma$.

This can be calculated as:

$$
p(\langle s, l\rangle)=p_{l}(l) \cdot p_{s}(\langle s, l\rangle) \approx \frac{p_{s}(\langle s, l\rangle)}{\left|G_{r e f}\right|}
$$

where $p_{l}(\sigma)$ is the probability a random $s$-mer begins at $\sigma$ 's offset, which we approximate by $\frac{1}{\left|G_{r e f}\right|} \cdot p_{s}(\sigma)$ is the probability a localized $s$-mer starting at $l$ has alleles matching $\sigma$ 's.

We approximate $p_{s}(\sigma)$ by assuming independence and multiplying the frequencies of each allele, or, if phasing information is available, by using allele co-occurrence frequencies.

Population coverage The population coverage $C(G)$ of an augmented reference $G$ is proportional to the population variation included, weighted by allele frequency. Specifically:

$$
C(G)=\sum_{\langle s, l\rangle \in G} p(\langle s, l\rangle)
$$

Note that $C\left(G_{r e f}\right) \leq C(G) \leq C\left(G^{*}\right)=1$.

We want to prioritize alleles according to how much they increase $C(G)$. To do so accurately, each variant's effect on $C(G)$ must be calculated according to which nearby variants (within $s-1$ positions) are already in G. While this is possible, it requires much recalculation of scores as variants are added to $G$. It also means there is no way to produce a single, static list of per-variant model scores. For these reasons, we instead compute each variant's effect on $C(G)$ assuming that all surrounding variants are already in $G$; in other words, we compute the decrease in $C(G)$ caused by removing the variant from $G *$. We call this the complete graph assumption.

Although FORGe is capable of using phasing data-describing which alleles co-occur on the same haplotype-the complete graph assumption makes this irrelevant for our calculation here. We do make (optional) use of phasing data in the Hybrid model, discussed below.

Uniqueness The uniqueness $U(G)$ of a genome $G$ decreases as the multiplicities of its $k$-mers increase, i.e., as the genome becomes more repetitive. Let $f_{G}(s)$ be the number of $\left\langle s^{\prime}, l^{\prime}\right\rangle$-mers in genome $G$ with $s=s^{\prime}$. We define uniqueness of the genome as:

$$
U(G)=\sum_{\langle s, l\rangle \in G} \frac{1}{f_{G}(s)}
$$

Adding a variant to the genome can either increase or decrease $U(G)$. Specifically, an $\langle s, l\rangle$-mer overlapping the variant increases $U(G)$ if there is no other $\left\langle s^{\prime}, l^{\prime}\right\rangle$-mers with $s=s^{\prime}$. Alternately, an $\langle s, l\rangle$-mer overlapping the variant decreases $U(G)$ if there are any other $\left\langle s^{\prime}, l^{\prime}\right\rangle$-mers with $s=s^{\prime}$.

While we rely on this definition below, we do not expect uniqueness alone to be an effective variant model. This is because for most variants all the added (overlapping) $\langle s, l\rangle$-mers are unique. All such variants therefore receive an identical score. The hybrid measure, presented next, effectively breaks ties by also considering allele frequency. 
Hybrid score The hybrid score $H(G)$ of a genome $G$ considers both population coverage and uniqueness.

Again let $f_{G}(s)$ be the number of $\left\langle s^{\prime}, l^{\prime}\right\rangle$-mers in $G$ with $s=s^{\prime}$ and let $p\left(\left\langle s^{\prime}, l^{\prime}\right\rangle\right)$ be the probability a random $\langle s, l\rangle$-mer drawn from a random individual equals $\left\langle s^{\prime}, l^{\prime}\right\rangle$. We define the hybrid measure $H(G)$ of an augmented reference $G$ as

$$
H(G)=\sum_{\langle s, l\rangle \in G} \frac{p(\langle s, l\rangle)}{f_{G}(s)}
$$

Note that this is simply the dot product of the terms from the $C(G)$ and $U(G)$ sums.

For a variant $v$, we wish to compute the increase in $H(G)$ caused by adding $v$. For each $\langle s, l\rangle$-mer overlapping $v$ and containing the alternate allele, let $\left\langle s, l_{1}\right\rangle,\left\langle s, l_{2}\right\rangle, \ldots,\left\langle s, l_{n}\right\rangle$ be all other $\langle s, l\rangle$-mers with the same sequence $s$. Before adding $v$, the hybrid score can be written as

$$
C+\sum_{i=1}^{n} \frac{p\left(\left\langle s, l_{i}\right\rangle\right)}{n}
$$

where $C$ is the hybrid-score portion due to the $\left\langle s^{\prime}, l\right\rangle$-mers with $s^{\prime} \neq s$. After adding $\langle s, l\rangle$ to $G$, the score becomes

$$
C+\sum_{i=1}^{n} \frac{p\left(\left\langle s, l_{i}\right\rangle\right)}{n+1}+\frac{p(\langle s, l\rangle)}{n+1}
$$

The change in hybrid score due to the addition of $\langle s, l\rangle$ is

$$
\begin{aligned}
\Delta H_{s, l} & =\sum_{i=1}^{n} \frac{p\left(\left\langle s, l_{i}\right\rangle\right)}{n+1}+\frac{p(\langle s, l\rangle)}{n+1}-\sum_{i=1}^{n} \frac{p\left(\left\langle s, l_{i}\right\rangle\right)}{n} \\
& =\frac{p(\langle s, l\rangle)-\frac{1}{n} \sum_{i=1}^{n} p\left(\left\langle s, l_{i}\right\rangle\right)}{n+1}
\end{aligned}
$$

Assuming each $\langle s, l\rangle$-mer overlapping variant $v$ has a distinct sequence $s$, their $\Delta H_{s, l}$ terms are independent. Thus the total change in hybrid score due to the addition of $v$ is the sum of the $\Delta H_{s, l}$ ls for each $\langle s, l\rangle$-mer overlapping and including $v$.

There are a couple caveats to how FORGe implements the hybrid model. First, As with the Pop Cov model, we make the complete graph assumption, allowing us to produce a scored variant list without dynamic re-scoring of variants as they are added.

Second, computing $\Delta H_{s, l}$ 's for all variants is expensive, since it involves calculating the read probability for each other occurrence of sequence $s$ for every overlapping $\langle s, l\rangle$-mer.

Instead, we approximate it using average probabilities. Specifically, we pre-calculate $\bar{p}_{\text {ref }}$, the average $p(\langle s, l\rangle)$ for all $\langle s, l\rangle$-mers in $G_{r e f}$, and $\bar{p}_{*}$, the average $p(\langle s, l\rangle)$ for all $\langle s, l\rangle$-mers in $G^{*}$ but not in $G_{r e f}$. We approximate the summation with a weighted average: $\frac{1}{n} \sum_{i=1}^{n} p\left(\left\langle s, l_{i}\right\rangle\right) \approx \frac{1}{f_{G^{*}}(s)}\left[\left(f_{G^{*}}(s)-f_{G_{r e f}}(s)\right) \cdot \bar{p}_{*}+f_{G_{r e f}}(s) \cdot \bar{p}_{r e f}\right]$

Whereas the complete graph assumption rendered phasing data irrelevant to the Pop Cov model, we can use phasing data in the Hybrid model. This is because the Hybrid model weights the terms of the sum according to their frequency in the genome. By default, FORGe uses phasing information when it is available.

Hybrid score implementation The Uniqueness and Hybrid models are concerned with $s$-mer counts both in the linear reference genome $\left(G_{r e f}\right)$ and in the complete augmented reference $\left(G^{*}\right)$. FORGe uses Jellyfish v2.2.6 [32] to calculate these counts. Since Jellyfish counts $s$-mers in a FASTA input file, FORGe must first construct an augmented FASTA such that $\langle s, l\rangle$-mers in this FASTA map one-to-one to $\langle s, l\rangle$-mers in $G^{*}$. This is also the goal of the Enhanced Reference Genome [43] representation, which accomplishes this by adding $2^{k}-1$ "enhanced segments" for every length-s window containing $k$ variants. Thus, to obtain $s$-mer counts for $G *$, we first constructed such as FASTA file using our implementation of the ERG, then counted $s$-mers using Jellyfish.

Once $s$-mers have been counted, FORGe computes the average probability for reads in the linear reference $\left(\bar{p}_{\text {ref }}\right)$ and in the complete augmented reference $\left(\bar{p}_{*}\right)$, for use in the Hybrid model formula. Finally, we compute the change in $H(G)$ for each $s$-mer in both and update the Hybrid model scores for every variant with an alternate allele in that read. After this, we have the full set of Hybrid model scores for all variants.

Considering blowup Adding variants to the augmented reference increases computational costs, including (a) size of the index on disk, (b) memory footprint during read alignment, and (c) time required for read alignment. We collectively refer to these as "blowup." Blowup is most drastic in genomic regions where variants are densely clustered, driving a exponential increase in the number of allelic combinations possible. A model based purely on minimizing blowup would prioritize isolated variants over those in clusters. We do not expect such a model to perform well on its own, though, since (like the Uniqueness model described above) it would fail to prioritize among the isolated variants. For this reason, we sought a way to combine a blowup avoidance strategy with the models already described above.

Selecting variants with blowup avoidance After ranking variants, FORGe selects the subset of variants to include in the augmented reference. The user specifies either a number or a fraction of all variants to include. 
In the simplest case, variants are chosen in order, starting with the highest-scoring variant, until the desired number have been included.

As an additional defense against blowup, we also propose a dynamic re-scoring scheme that can be added to an existing model. In this scheme, when a variant is added to the reference, FORGe searches for other variants within $s$ nt (the window length) of the added variant that have not yet been selected for addition. These nearby variants are re-scored by multiplying their score by a penalty factor $w$, where $0<w \leq 1$. By letting $w$ be variable, FORGe can trade off between maximizing the model score and minimizing blowup. $w=1$ maintains the original scores, whereas a penalty near $w=0$ would ensure all isolated variants were added before any neighboring variants. We found that a penalty of $w=0.5$ performed well in practice, and this is FORGe's default, used in all experiments performed here. Pop Cov+ and Hybrid + are how we refer to those models when they are combined with this dynamic re-scoring scheme.

Breaking ties Variants can be given identical scores using these models. For instance, variants with the same allele frequency will receive the same score by the Pop Cov model. These ties are broken according to the variants' position on the genome. We define variant $A$ to be upstream of (and higher priority than) variant $B$ if it is on a lower-numbered chromosome or if its offset is to the left of B's.

\section{Discussion}

FORGe's modeling of positive and negative effects of including genetic variants in an augmented reference yields accuracy-blowup tradeoffs superior to current approaches. We proposed models for prioritizing variants with distinct rationales and strengths. We found repeatedly that the most advantageous set of variants consisted of a fraction (6-30\%) of the variants called in the 1000 Genomes project. This was true across a variety of alignment scenarios: for two different graph alignment methods (HISAT2 and ERG), for both unpaired and paired-end alignment modes, when just SNVs and when both SNVs and indels are included, for both a single human chromosome and for the whole human genome, and for both CEPH and YRI individuals. We also showed that FORGe's modeling can substantially improve downstream results related to reference bias and HLA typing, also at relatively low variants-inclusion fractions.

To test if FORGe's results yield a simple filtering rule, we can translate the peak-performing variant inclusion fractions for the Pop Cov model into allele frequency thresholds. For the Chromosome-9 NA12878 experiments, the $8 \%$ variant inclusion fraction performed best in both the unpaired (Fig. 1) and paired-end (Additional file 1:
Figure S3) experiments. This translates to an allele frequency threshold of $\geq 7.42 \%$. For the Chromosome- 9 YRI experiment (Additional file 1: Figure S4), the bestperforming fraction of $10 \%$ translated to an allele frequency threshold of $\geq 3.76 \%$. While these fall on either side of the $5 \%$ threshold used in at least one prior study [31], further work is needed to establish whether that or any other simple threshold is justifiable in general. A finer-grained sweep over variant inclusion fractions would yield a sharper threshold, for example. For now, our strategy of gradually introducing variants in the context of a simulation study is both principled and practical.

FORGe and HISAT2 combine to make a practical graph aligner that works with human data with large variant databases like the 1000 Genomes Phase 3 call set. Using hisat2-build to index a GRCh37-based graph genome with the top $8 \%$ of variants from Phase- 3 set required $4 \mathrm{~h}$ and $165 \mathrm{~GB}$ of memory. Aligning 20 million reads to this graph required $19 \mathrm{~min}$ and $6.5 \mathrm{~GB}$ of memory, about 50\% more time and 50\% more memory than HISAT2 requires to align to the linear GRCh37 genome. (To prioritize the variants prior to indexing, FORGe required about 110 min on a single processor.) This is competitive with the performance of aligners like Bowtie 2 and BWA-MEM when aligning to the linear reference, suggesting graph-based tools are ready for broader use.

Though we estimate that the overall improvement in alignment accuracy for a $40 \times$ whole-genome DNA sequencing experiment would lead to $4.8 \mathrm{M}$ more correctly aligned reads and $1.2 \mathrm{M}$ fewer incorrectly aligned reads, the magnitude of the improvement imparted by modeling variants depends on the genomic region. For some regions and variant classes (rare, isolated SNVs), the benefit is small.

To improve alignment to these regions might require an iterative approach that aligns to a graph containing known variants, calls donor-specific variants, then realigns to a graph that includes both. Strategies like this are implemented in the GATK HaplotypeCaller [10], GraphTyper [15] and other tools [35]. Better variant models might also benefit these hard cases. Even so, the effects we measured translate into substantial net increases in the number of correctly aligned reads, and the results are pronounced in regions such as MHC as shown by Additional file 1: Figures S6 and S9.

An ethnicity-specific reference conferred a slight accuracy improvement compared to a pan-ethnic reference with a similar number of variants. This is notable in light of proposals to use ethnicity-specific references $[2,33]$. It suggests that the advantages of an inclusive reference, applicable regardless of the donor individual's ethnicity, might outweigh the slight accuracy gain that comes with ethnicity-specificity. Also, ethnicity-specific references 
could be counterproductive or misleading in cases where donor ethnicity is reported incorrectly or where the donor is admixed [19].

The accuracy achieved at relatively small fractions of the 1000 Genomes variants has implications for the design of graph aligners. A central challenge for these tools is to operate efficiently even when variants are densely clustered, causing local explosion in the number of allelic combinations. But our observations that peak accuracy occurs at a relatively small fraction of variants, and that memory footprint increases by a factor of 2 or less at peak accuracy, suggests that this is not a major barrier to practical graph-genome alignment as long as variants are chosen carefully.

It should also be possible to adapt FORGe to study how including structural variants can improve alignment. A common observation of studies that have assembled human genomes from long reads is that the assemblies contain many megabases of sequence not present in the standard human reference [2, 5, 45, 47]. The models we propose are equally applicable to structural variants, assuming the variants are called in enough individuals to estimate allele frequencies accurately.

While we primarily investigated unpaired alignment here, we also showed that the chromosome-9 results generalized to paired-end alignment (Additional file 1: Figure S3). Generally speaking, this work can be adapted to paired-end alignment, with the main issue being how to adjust the method's window lengths as a function of the paired-end dataset's read and fragment lengths. The windows in question are (a) the $s$-mer length used in the model, (b) the maximum window length used when forming enhanced segments for ERG-based alignment, and (c) for blowup avoidance in the Pop Cov+ and Hybrid + models, the radius to look within when seeking nearby variants to deprioritize. While one option is to simply increase the window size to the maximum fragment length, that can easily lead to an unacceptable blowup penalty. For this reason, we suspect that there is no practical way to adapt our ERG-based to paired-end alignment; rather, as we explain, we think it is best viewed as a model for the seed-finding step of a seed-and-extend aligner that might itself handle paired ends. Initial experiments suggest that it is practical to leave the window lengths relatively short-the length of a read rather than a fragment-when using HISAT2 for paired-end alignment (Additional file 1: Figure S3). Further exploration is needed to more fully characterize the relationship between FORGe window length and fragment and read lengths for paired-end reads.

While we found that including FORGe-selected variants improved results for a downstream HLA-typing method, we also found the method failed to achieve perfect accuracy based only on HISAT2+FORGe's alignments. This was likely because one of NA12878's HLA alleles (DQB1*02:01) was so different from the referenceeven after including the FORGe-selected variants-that its identifying reads failed to align (see Additional file 1 : Note S7). This highlights the continued importance of other alignment methods that use alternate assemblies such as the GRCh38 ALT loci [7] or that can align to graphs that include larger-scale variation [18] in addition to relatively small variants studied here and in the 1000 Genomes Project.

\section{Additional file}

Additional file 1: Supplementary information. Contains Notes S1-S7 and Figures S1-S9. (PDF 620 kb)

\section{Acknowledgements}

We thank Daehwan Kim and Michael Schatz for their helpful comments on a draft of this manuscript.

\section{Funding}

JP and BL were supported by National Science Foundation grant IIS-1349906 and National Institutes of Health/National Institute of General Medical Sciences grant R01GM118568.

\section{Availability of data and materials}

The FORGe software is available at https://github.com/langmead-lab/FORGe/ releases/tag/v1.1 under the open source MIT license. It is written in Python and was tested on Linux and Mac operating systems. An archival version of the software evaluated here is at https://doi.org/10.5281/zenodo.1482926 [40]. The FORGe repo also includes our implementation of the Enhanced Reference Genome, originally proposed by Satya et al. [43]. The experiments described in this paper are open source under the open source MIT license and available at https://github.com/langmead-lab/FORGe-experiments. An archival version of this software is available at doi: 10.5281/zenodo.1482928 [42]. We provide three variant datasets and HISAT2 indexes, available at ftp://ftp.ccb.jhu.edu/pub/langmead/forge and at the listed DOls. These include the optimal graph genomes described above for chromosome 9 using only SNVs (NA12878_chr9 subdirectory or https://doi.org/10.6084/m9. figshare.7325831 [38]), for chromosome 9 using SNVs and indels

(NA12878_chr9_indels subdirectory or https://doi.org/10.6084/m9. figshare.7325837 [37]), and for the full genome (NA12878_full_genome subdirectory or https://doi.org/10.6084/m9.figshare.7325840 [39]), all with NA12878 and family members withheld. We also provide what we consider the best full genome graph, derived from all 1000 Genomes SNVs and indels from all individuals and all populations using the Pop Cov+ strategy and taking the top 10\% of variants (best_full_genome subdirectory or https://doi. org/10.6084/m9.figshare.7327016 [41]). The variants and index files in the (best_full_genome subdirectory) are the ones we suggest others use for future studies.

\section{Authors' contributions}

$\mathrm{JP}$ and $\mathrm{BL}$ designed the method and wrote the software. NC designed and executed the HLA typing experiment. JP, BL, and NC wrote the manuscript. All authors read and approved the final manuscript.

Ethics approval and consent to participate Not applicable.

\section{Consent for publication}

Not applicable.

\section{Competing interests}

The authors declare that they have no competing interests.

\section{Publisher's Note}

Springer Nature remains neutral with regard to jurisdictional claims in published maps and institutional affiliations. 
Received: 12 May 2018 Accepted: 26 November 2018 Published online: 17 December 2018

\section{References}

1. Altshuler DM, Gibbs RA, Peltonen L, Altshuler DM, Gibbs RA, Peltonen L, Dermitzakis E, Schaffner SF, Yu F, Peltonen L, Dermitzakis E, Bonnen PE, Altshuler DM, Gibbs RA, de BakkerPI, Deloukas P, Gabriel SB, Gwilliam R, Hunt S, Inouye M, Jia X, Palotie A, Parkin M, Whittaker P, Yu F, Chang K, Hawes A, Lewis LR, Ren Y, Wheeler D, Gibbs RA, Muzny DM, Barnes C, Darvishi K, Hurles M, Korn JM, Kristiansson K, Lee C, McCarrol SA, Nemesh J, Dermitzakis E, Keinan A, Montgomery SB, Pollack S, Price AL, Soranzo N, Bonnen PE, Gibbs RA, Gonzaga-Jauregui C, Keinan A, Price AL, Yu F, Anttila V, Brodeur W, Daly MJ, Leslie S, McVean G, Moutsianas L, Nguyen H, Schaffner SF, Zhang Q, Ghori MJ, McGinnis R, McLaren W, Pollack S, Price AL, Schaffner SF, Takeuchi F, Grossman SR, Shlyakhter I, Hostetter EB, Sabeti PC, Adebamowo CA, Foster MW, Gordon DR, Licinio J, Manca MC, Marshall PA, Matsuda I, Ngare D, Wang VO, Reddy D, Rotimi CN, Royal CD, Sharp RR, Zeng C, Brooks LD, McEwen JE. Integrating common and rare genetic variation in diverse human populations. Nature. 2010;467(7311):52-8.

2. Ameur A, Che H, Martin M, Bunikis I, Dahlberg J, Höijer I, Häggqvist S Vezzi F, Nordlund J, Olason P, Feuk L, Gyllensten U. De novo assembly of two swedish genomes reveals missing segments from the human grch38 reference and improves variant calling of population-scale sequencing data. 2018.

3. Auton AEA. A global reference for human genetic variation. Nature. 2015;526(7571):68-74.

4. Brandt DY, Aguiar VR, Bitarello BD, Nunes K, Goudet J, Meyer D. Mapping bias overestimates reference allele frequencies at the HLA Genes in the 1000 Genomes Project Phase I Data. G3 (Bethesda). 2015;5(5):931-41.

5. Chaisson MJ, Huddleston J, Dennis MY, Sudmant PH, Malig M, Hormozdiari F, Antonacci F, Surti U, Sandstrom R, Boitano M, Landolin JM, Stamatoyannopoulos JA, Hunkapiller MW, Korlach J, Eichler EE. Resolving the complexity of the human genome using single-molecule sequencing. Nature. 2015;517(7536):608-11.

6. Church DM, Schneider VA, Graves T, Auger K, Cunningham F, Bouk N, Chen HC, Agarwala R, McLaren WM, Ritchie GR, Albracht D, Kremitzki M, Rock S, Kotkiewicz H, Kremitzki C, Wollam A, Trani L, Fulton L, Fulton R, Matthews L, Whitehead S, Chow W, Torrance J, Dunn M, Harden G, Threadgold G, Wood J, Collins J, Heath P, Griffiths G, Pelan S, Grafham D, Eichler EE, Weinstock G, Mardis ER, Wilson RK, Howe K, Flicek P, Hubbard T. Modernizing reference genome assemblies. PLoS Biol. 2011;9(7):e1001091.

7. Church DM, Schneider VA, Steinberg KM, Schatz MC, Quinlan AR, Chin CS, Kitts PA, Aken B, Marth GT, Hoffman MM, Herrero J, Mendoza ML, Durbin R, Flicek P. Extending reference assembly models. Genome Biol. 2015;16:13.

8. Danek A, Deorowicz S, Grabowski S. Indexes of large genome collections on a PC. PLOS ONE. 2014;9(10):e109384.

9. Degner JF, Marioni JC, Pai AA, Pickrell JK, Nkadori E, Gilad Y, Pritchard JK. Effect of read-mapping biases on detecting allele-specific expression from RNA-sequencing data. Bioinformatics. 2009;25(24):3207-12.

10. DePristo MA, Banks E, Poplin R, Garimella KV, Maguire JR, Hartl C, Philippakis AA, del Angel G, Rivas MA, Hanna M, McKenna A, Fennell TJ, Kernytsky AM, Sivachenko AY, Cibulskis K, Gabriel SB, Altshuler D, Daly MJ. A framework for variation discovery and genotyping using next-generation DNA sequencing data. Nat Genet. 2011;43(5):491-8.

11. Dewey FE, Chen R, Cordero SP, Ormond KE, Caleshu C, Karczewski KJ, Whirl-Carrillo M, Wheeler MT, Dudley JT, Byrnes JK, Cornejo OE, Knowles JW, Woon M, Sangkuhl K, Gong L, Thorn CF, Hebert JM, Capriotti E, David SP, Pavlovic A, West A, Thakuria JV, Ball MP, Zaranek AW, Rehm HL, Church GM, West JS, Bustamante CD, Snyder M, Altman RB, Klein TE, Butte AJ, Ashley EA. Phased whole-genome genetic risk in a family quartet using a major allele reference sequence. PLoS Genet. 2011;7(9):e1002280.

12. Dilthey A, Cox C, Iqbal Z, Nelson MR, McVean G. Improved genome inference in the $\mathrm{MHC}$ using a population reference graph. Nat Genet. 2015;47(6):682-8.

13. Dolgalev I, Sedlazeck F, Busby B. DangerTrack: a scoring system to detect difficult-to-assess regions. F1000Res. 2017;6:443.

14. Eberle MA, Fritzilas E, Krusche P, Kallberg M, Moore BL, Bekritsky MA, lqbal Z, Chuang HY, Humphray SJ, Halpern AL, Kruglyak S, Margulies EH,
McVean G, Bentley DR. A reference data set of 5.4 million phased human variants validated by genetic inheritance from sequencing a three-generation 17-member pedigree. Genome Res. 2017;27(1):157-64.

15. Eggertsson HP, Jonsson $H$, Kristmundsdottir $S$, Hjartarson $E$, Kehr $B$, Masson G, Zink F, Hjorleifsson KE, Jonasdottir A, Jonasdottir A, Jonsdottir I, Gudbjartsson DF, Melsted P, Stefansson K, Halldorsson BV. Graphtyper enables population-scale genotyping using pangenome graphs. Nat Genet. 2017;49(11):1654-60.

16. Ferragina P, Manzini G. Opportunistic data structures with applications In: Foundations of Computer Science, 2000. Proceedings. 41st Annual Symposium on. IEEE; 2000. p. 390-398.

17. Gan X, Stegle O, Behr J, Steffen JG, Drewe P, Hildebrand KL, Lyngsoe R, Schultheiss SJ, Osborne EJ, Sreedharan VT, Kahles A, Bohnert R, Jean G, Derwent P, Kersey P, Belfield EJ, Harberd NP, Kemen E, Toomajian C, Kover PX, Clark RM, Ratsch G, Mott R. Multiple reference genomes and transcriptomes for Arabidopsis thaliana. Nature. 2011;477(7365):419-23.

18. Garrison E, Siren J, Novak AM, Hickey G, Eizenga JM, Dawson ET, Jones W, Garg S, Markello C, Lin MF, Paten B, Durbin R. Variation graph toolkit improves read mapping by representing genetic variation in the reference. Nat Biotechnol. 2018;36(9):875-9.

19. Genovese G, Handsaker RE, Li H, Altemose N, Lindgren AM, Chambert K, Pasaniuc B, Price AL, Reich D, Morton CC, Pollak MR, Wilson JG, McCarroll SA. Using population admixture to help complete maps of the human genome. Nat Genet. 2013;45(4):406-14.

20. Holtgrewe M. Mason-a read simulator for second generation sequencing data. Technical Report FU Berlin. 2010.

21. Huang L, Popic V, Batzoglou S. Short read alignment with populations of genomes. Bioinformatics. 2013;29(13):i361-370.

22. Iqbal Z, Caccamo M, Turner I, Flicek P, McVean G. De novo assembly and genotyping of variants using colored de Bruijn graphs. Nat Genet. 2012;44(2):226-32.

23. Karthikeyan S, Bawa PS, Srinivasan S. hg19K: addressing a significant lacuna in hg19-based variant calling. Mol Genet Genomic Med. 2017;5(1): 15-20.

24. Kim D, Langmead B, Salzberg SL. HISAT: a fast spliced aligner with low memory requirements. Nat Methods. 2015;12(4):357-60.

25. Kim D, Paggi J, Salzberg S. Hisat-genotype: next generation genomic analysis platform on a personal computer. bioRxiv. 2018.

26. Langmead B. A tandem simulation framework for predicting mapping quality. Genome Biol. 2017;18(1):152.

27. Langmead B, Trapnell C, Pop M, Salzberg SL. Ultrafast and memory-efficient alignment of short DNA sequences to the human genome. Genome Biol. 2009;10(3):R25.

28. Lee $H$, Kingsford C. Kourami: graph-guided assembly for novel human leukocyte antigen allele discovery. Genome Biol. 2018;19(1):16.

29. Li H, Ruan J, Durbin R. Mapping short DNA sequencing reads and calling variants using mapping quality scores. Genome Res. 2008;18(11):1851-8.

30. Liu B, Guo H, Brudno M, Wang Y. deBGA: read alignment with de Bruijn graph-based seed and extension. Bioinformatics. 2016;32(21):3224-32.

31. Maciuca S, del Ojo Elias C, McVean G, Iqbal Z. A natural encoding of genetic variation in a burrows-wheeler transform to enable mapping and genome inference. In: International Workshop on Algorithms in Bioinformatics. Springer; 2016. p. 222-33.

32. Marcais $G$, Kingsford C. A fast, lock-free approach for efficient parallel counting of occurrences of k-mers. Bioinformatics. 2011;27(6):764-70.

33. Maretty L, Jensen JM, Petersen B, Sibbesen JA, Liu S, Villesen P, Skov L, Belling K, Theil Have C, Izarzugaza JMG, Grosjean M, Bork-Jensen J, Grove J, Als TD, Huang S, Chang Y, Xu R, Ye W, Rao J, Guo X, Sun J, Cao H, Ye C, van Beusekom J, Espeseth T, Flindt E, Friborg RM, Halager AE, Le Hellard S, Hultman CM, Lescai F, Li S, Lund O, L?ngren P, Mailund T, Matey-Hernandez ML, Mors O, Pedersen CNS, Sicheritz-Ponten T, Sullivan P, Syed A, Westergaard D, Yadav R, Li N, Xu X, Hansen T, Krogh A, Bolund L, S?rensen TIA, Pedersen O, Gupta R, Rasmussen S, Besenbacher S, B? rglum AD, Wang J, Eiberg H, Kristiansen K, Brunak S, Schierup $\mathrm{MH}$. Sequencing and de novo assembly of 150 genomes from Denmark as a population reference. Nature. 2017;548(7665):87-91.

34. Novak AM, Hickey G, Garrison E, Blum S, Connelly A, Dilthey A, Eizenga J, Elmohamed MAS, Guthrie S, Kahles A, Keenan S, Kelleher J, Kural D, Li H, Lin MF, Miga K, Ouyang N, Rakocevic G, Smuga-Otto M, Zaranek AW, Durbin R, McVean G, Haussler D, Paten B. Genome graphs. BioRxiv. 2017. 
35. Otto TD, Sanders M, Berriman M, Newbold C. Iterative Correction of Reference Nucleotides (iCORN) using second generation sequencing technology. Bioinformatics. 2010;26(14):1704-7.

36. Paten B, Novak AM, Eizenga JM, Garrison E. Genome graphs and the evolution of genome inference. Genome Res. 2017;27(5):665-76.

37. Pritt J, Chen N, Langmead B. Best human chromosome 9 index with both snvs and indels used in forge study. 2018a. figshare. https://doi.org/ 10.6084/m9.figshare.7325837.

38. Pritt J, Chen N, Langmead B. Best human chromosome 9 index with snvs used in forge study. 2018b. figshare. https://doi.org/10.6084/m9.figshare. 7325831.

39. Pritt J, Chen N, Langmead B. Best whole-human index with both snvs and indels used in forge study. 2018c. figshare. https://doi.org/10.6084/ m9.figshare.7325840.

40. Pritt J, Chen N, Langmead B. Forge software. 2018d. Zenodo. https://doi. org/10.5281/zenodo.1482926.

41. Pritt J, Chen N, Langmead B. Recommended whole-human hisat2 index including top $10 \%$ of variants according to Pop Cov+ model. $2018 \mathrm{e}$. figshare. https://doi.org/10.6084/m9.figshare.7327016.

42. Pritt J, Chen N, Langmead B. Software for forge study experiments, 2018f. Zenodo. https://doi.org/10.5281/zenodo.1482928.

43. Satya RV, Zavaljevski N, Reifman J. A new strategy to reduce allelic bias in RNA-Seq readmapping. Nucleic Acids Res. 2012;40(16):e127.

44. Schneeberger K, Hagmann J, Ossowski S, Warthmann N, Gesing S, Kohlbacher O, Weigel D. Simultaneous alignment of short reads against multiple genomes. Genome Biol. 2009;10(9):R98.

45. Seo JS, Rhie A, Kim J, Lee S, Sohn MH, Kim CU, Hastie A, Cao H, Yun JY, Kim J, Kuk J, Park GH, Kim J, Ryu H, Kim J, Roh M, Baek J, Hunkapiller MW, Korlach J, Shin JY, Kim C. De novo assembly and phasing of a Korean human genome. Nature. 2016;538(7624):243-7.

46. Sherry ST, Ward MH, Kholodov M, Baker J, Phan L, Smigielski EM, Sirotkin K. dbSNP: the NCBI database of genetic variation. Nucleic Acids Res. 2001;29(1):308-11.

47. Shi L, Guo Y, Dong C, Huddleston J, Yang H, Han X, Fu A, Li Q, Li N, Gong S, Lintner KE, Ding Q, Wang Z, Hu J, Wang D, Wang F, Wang L, Lyon GJ, Guan Y, Shen Y, Evgrafov OV, Knowles JA, Thibaud-Nissen F, Schneider V, Yu CY, Zhou L, Eichler EE, So KF, Wang K. Long-read sequencing and de novo assembly of a Chinese genome. Nat Commun. 2016;7:12065

48. Sirén J. Indexing variation graphs. In: 2017 Proceedings of the Ninteenth Workshop on Algorithm Engineering and Experiments (ALENEX). SIAM; 2017. p. 13-27.

49. Sirén J, Välimäki N, Mäkinen V. Indexing graphs for path queries with applications in genome research. IEEE/ACM Trans Comput Biol Bioinforma. 2014;11(2):375-88. Barcelona, Spain.

50. The Computational Pan-Genomics Consortium. Computational pan-genomics: status, promises and challenges. Brief Bioinformatics. 2016

51. Thorvaldsdottir H, Robinson JT, Mesirov JP. Integrative Genomics Viewer (IGV): high-performance genomics data visualization and exploration. Brief Bioinformatics. 2013;14(2):178-92.

52. Walter KEA. The UK10K project identifies rare variants in health and disease. Nature. 2015;526(7571):82-90.

Ready to submit your research? Choose BMC and benefit from:

- fast, convenient online submission

- thorough peer review by experienced researchers in your field

- rapid publication on acceptance

- support for research data, including large and complex data types

- gold Open Access which fosters wider collaboration and increased citations

- maximum visibility for your research: over 100M website views per year

At $\mathrm{BMC}$, research is always in progress.

Learn more biomedcentral.com/submissions 